\title{
Decay widths at the scission point in nuclear fission
}

\author{
G. F. Bertsch $\odot^{*}$ \\ Institute for Nuclear Theory and Department of Physics, University of Washington, Seattle, Washington 98195, USA \\ L. M. Robledo ${ }^{\dagger}$ \\ Departamento de Física Teórica, Universidad Autónoma de Madrid, E-28049 Madrid, Spain \\ and Center for Computational Simulation, Universidad Politécnica de Madrid, Campus de Montegancedo, Boadilla del Monte, \\ 28660-Madrid, Spain
}

(Received 14 August 2019; published 11 October 2019)

\begin{abstract}
An outstanding problem in the theory of nuclear fission is understanding the Hamiltonian dynamics at the scission point. Here we apply the generator coordinate method to calculate decay widths for prescission configurations into the two-fragment continuum. Transitions that are allowed under diabatic dynamics can have widths up to several MeV. For nondiabatic decays through the pairing interaction, typical widths to a specific final-state channel are two to three orders of magnitude smaller. The nucleus ${ }^{236} \mathrm{U}$ is taken as a representative example in the calculations.
\end{abstract}

DOI: 10.1103/PhysRevC.100.044606

\section{INTRODUCTION}

The final step in nuclear fission is the rupture of the neck between the two nascent fragments, leaving them to interact only through long-range potential fields. The quantum dynamics of this scission process is quite complex and has resisted a satisfactory description within many-body Hamiltonian theory-see Ref. [1] for a recent review. Here we attempt to construct a fully quantum mechanical treatment in the framework of self-consistent mean-field theory following the formulation of Ref. [2]. The configuration space is constructed by the generator coordinate method $^{1}$ (GCM) and interactions are computed at the nucleon-nucleon level. Ideally, the GCM basis would be separated into configurations that are bound under mean-field dynamics and those that will evolve to separated postfission fragments under a mean-field Hamiltonian. The goal of this paper is to make some first estimates of the transitions between bound states and continuum channels defined in the same framework by chains of GCM configurations. The GCM constraints on the configurations are their $K$ partitions and the expectation value of a single-particle operator measuring the elongation of the system. An important practical question is the spacing of the configurations with respect to elongation in the chain representing a continuum channel. In Ref. [2] the quality of the paths was assessed by the overlaps of the configurations along the chain, but Hamiltonian dynamics of their interactions was left for the present exploratory study. We will also make use of Ref. [5] where the Hamiltonian dynamics of the GCM configurations leading to separated subsystems was treated in a general way.

\footnotetext{
*bertsch@uw.edu

†luis.robledo@uam.es

${ }^{1}$ See Refs. [3,4] for reviews of the method.
}

The transition rate of a bound configuration may be estimated by the envelope of its strength function in the eigenstates of the Hamiltonian in a basis including both the initial state and the chain of configurations representing the continuum. For weak coupling between the initial state and the continuum states, the decay width can be estimated by the Fermi golden rule (FGR),

$$
\Gamma=2 \pi|\langle i|H| f\rangle|^{2} \frac{d n_{f}}{d E} .
$$

Here $|f\rangle$ is a continuum wave function at the same energy as the initial-state $|i\rangle$ and $d n_{f} / d E$ is the final-state density of states.

The separation between bound states and those in a continuum-connected chain is facilitated by using a basis of Hartree-Fock wave functions in axially symmetric mean fields. This permits GCM constraints on the occupation numbers in the wave function, the $K$ partition mentioned above, as well as the familiar shape constraints. The continuumconnected chain of GCM configurations is constructed in a diabatic approximation, namely conserving the $K$ partition along the chain. However, the separation between the two kinds of wave function is not perfect, due in part to the nonorthogonality of GCM basis states. Also, as seen in Ref. [2], scission might require a considerable reorganization of the many-body wave function even within a given channel. In that case, one might want to treat the prescission side of the chain as the decaying state. In such cases where the initial state is not orthogonal to the continuum channel configurations it is necessary to orthogonalize the wave functions before applying the FGR. The method used here is via the Lanczos-basis strength function [6] as described in the Appendix. 
In the next section, we present the methodology to construct continuum final-state wave functions in the GCM. In Sec. III we apply the method to a continuum channel in the fission of ${ }^{236} \mathrm{U}$, partially following the formulation in Ref. [5]. Section IV presents three examples of calculated decay widths to that channel. We find that the decays can vary over many orders of magnitude, depending in part whether the configurations are diabatically connected or not. In the last section we discuss possible improvements of the methodology and the application to physical observables.

\section{METHODOLOGY}

The first task in applying the FGR to decay widths is to build multiconfiguration wave functions representing the decay channels. The general approach and some numerical considerations are discussed in Ref. [5]. The configurations are defined as the Hartree-Fock mean-field states obtained by the GCM based on axially symmetric mean fields. The axial symmetry permits good $K$ quantum numbers, thus giving the partition by a set of quantum numbers for the many-particle configurations. In addition, the configurations are labeled by a set of density constraints as part of the GCM representation. In previous work we used the mass quadrupole operator $Q_{2}=$ $z^{2}-\left(x^{2}+y^{2}\right) / 2$ to generate a coordinate for the fission path. Here we will use instead the relative distance between the two nascent fragments

$$
z_{\text {rel }}=\left(z-z_{0}\right) \Theta\left(z-z_{0}\right) / A_{R}+\left(z_{0}-z\right) \Theta\left(z_{0}-z\right) / A_{L},
$$

where $A_{R}, A_{L}$ are the number of particles on each side and $z_{0}$ is the longitudinal position of the dividing plane between the two nascent fragments. This field has the advantage that it is exactly the coordinate needed for the continuum twofragment wave function of the final state. Another benefit is that the nuclear part of the force along that coordinate can be calculated from the properties of the wave function on the dividing plane [7].

The disadvantage of using Eq. (2) is that it requires two parameters, the dividing plane at $z=z_{0}$ between the two nascent fragments and the masses on each side of the dividing plane $A_{L}$ and $A_{R}$. We determine these from the density distribution immediately before scission. In particular, $z_{0}$ is taken as the point where the density on the $z$ axis $\rho(x=0, y=0, z)$ is minimum. Besides $z_{\text {rel }}$, we shall also employ the neck-size operator [8]

$$
\hat{N}_{\text {neck }}=\sum_{i=1}^{A} e^{-\left(z_{i}-z_{0}\right)^{2} / a^{2}}
$$

to distinguish pre- and postscission configurations. The wave functions are computed using the code HFBaxial [9], which produces constrained wave functions in a Hartree-FockBogoliubov (HFB) framework. Here we make use of a technical device to force the wave functions toward a Hartree-Fock (HF) limit. Namely, an additional constraint is applied to the fluctuation in proton and neutron particle numbers. Taking the constraint as

$$
\left\langle(\hat{N}-\langle\hat{N}\rangle)^{2}\right\rangle=0.1
$$

produces HFB wave functions that are close to HF configurations. The Gogny D1S energy functional is used in this work; other details are the same as in Ref. [2].

The actual construction of the multiconfiguration eigenstates in the space of GCM configurations is quite straightforward, given the matrix elements of the overlap matrix $S$ and a Hamiltonian matrix $H$ between configurations

$$
\begin{aligned}
S_{i j} & =\langle i \mid j\rangle, \\
H_{i j} & =\langle i|\mathcal{E}| j\rangle,
\end{aligned}
$$

where $|i\rangle$ and $|j\rangle$ are GCM configurations in the space. The operator $\mathcal{E}$ is the energy density functional (EFT), treated here as a Hamiltonian. This runs into well-known difficulties for interactions in the EFT that have fractional powers of the density [10-12]; we follow common practice here and compute these matrix elements using the "mixed-density" prescription ${ }^{2}$ [13], Eq. (C.3)]. The code GCMaxial [9] is used to compute the $S$ and $H$ matrices from the wave functions generated by HFBaxial. The code and other details are available in the Supplemental Material [14]. The Hamiltonian dynamics is governed by the equation

$$
i S \frac{d}{d t} \Psi(t)=H \Psi(t)
$$

the eigenvalue equation is the same with $i d / d t$ replaced by the eigenvalue.

There are two crucial assumptions in our procedure for constructing continuum channels in the GCM framework. The first is that the two-fragment final-state wave function factorizes into products of center-of-mass and internal wave functions, and the second is that the center-of-mass wave functions are Gaussian. Then the overlaps of final-state configurations can be expressed

$$
\left\langle z_{1} \mid z_{2}\right\rangle=\exp \left[-\left(z_{1}-z_{2}\right)^{2} / 4 s^{2}\right],
$$

where $s$ is the size parameter in the Gaussian relativecoordinate wave function $\psi$ :

$$
\psi_{n}\left(z_{\text {rel }}\right)=\frac{1}{s^{1 / 2} \pi^{1 / 4}} \exp \left[-\left(z_{\text {rel }}-z_{n}\right)^{2} / 4 s^{2}\right] .
$$

In Ref. [5] we analyzed the accuracy of the generated continuum wave functions for simple model Hamiltonians. The set of GCM configurations of given $K$ partition form a chain with respect to $\left\langle z_{\text {rel }}\right\rangle$. A useful measure in constructing the chain is the overlap distance $\zeta$ between configurations on the chain. For a chain segment containing $N$ configuration the overlap distance between the two end links is defined

$$
\zeta_{1, N}=\sum_{n=1}^{N-1}(-\log |\langle n \mid n+1\rangle|)^{1 / 2},
$$

where $\langle n \mid n+1\rangle$ are the overlaps of adjacent configurations. This definition has the advantage that it is insensitive to the

\footnotetext{
${ }^{2}$ An important consideration for our purposes is that the internal energy of a state with two separated nuclei is the sum of the individual energies.
} 
number of intermediate links and their spacing. This property is rigorously true for Gaussian overlaps.

An important consideration is how closely to space members of the chain. We found that reasonably accurate representations of the GCM Hamiltonian could be constructed with nearest-neighbor overlaps in the range $\langle n \mid n+1\rangle \approx 0.3-0.7$, corresponding to overlap distances $\zeta_{n, n+1} \approx 0.6-1.1$.

One caveat is that the GCM representation as constrained here has only a limited capability to approximate finitemomentum states. The controlling parameter is the kinetic energy and its spread associated with the Gaussian wave packet in the relative coordinate. Without explicit momentum constraints, the excitation energies that can be treated are of order

$$
E \sim \frac{\hbar^{2}}{2 M_{\mathrm{red}} s^{2}}
$$

or less. Here $M_{\text {red }}$ is the reduced mass associated with the relative coordinate. The problem is serious for fission because the Coulomb field in the final state causes a large variation in the energies of the configurations as a function of the separation $r_{\text {rel }}$.

In this work we deal with the problem by modifying the $S$ and $H$ matrix elements associated with the separated fragments to simulate a flat-bottomed potential in $z_{\text {rel }}$. This is implemented as follows. Partial $S$ and $H$ matrices representing prescission and the closest postscission configurations are computed in the usual way. Those matrices are embedding as the first blocks in larger matrices $S^{\prime \prime}$ and $H^{\prime \prime}$ with the additional entries representing the more distant postscission configurations. The additional elements in $S^{\prime \prime}$ are determined iteratively as

$$
S_{i, j}^{\prime \prime}=\exp \left[-\left(\zeta_{i, j-1}+\zeta_{j-1, j}\right)^{2}\right]
$$

where $j$ is an added state, $\zeta_{j-1, j}$ is its assumed distance from the previous state, and $i \leqslant j-1$. The treatment of the Hamiltonian matrix element is more complicated. The diagonal matrix elements are taken as $H_{j, j}^{\prime \prime}=H_{k, k}$, where $k$ is the last configuration included in the full GCM. The off-diagonal ones need to take into account the contributions due to nonzero overlaps. The intrinsic contribution is modeled by a quadratic

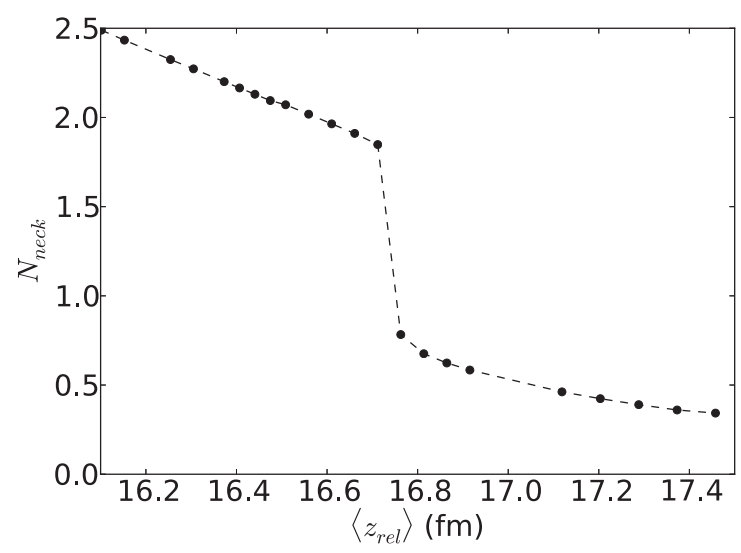

FIG. 1. Neck size $N_{\text {neck }}$ [Eq. (3)] of Glider configurations constrained by the $z_{\text {rel }}$ operator [Eq. (3)].

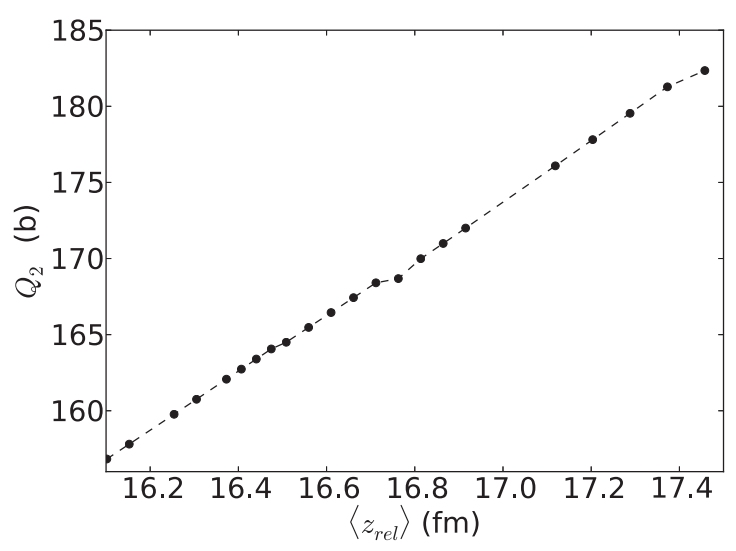

FIG. 2. Mass quadrupole moments $Q_{2}$ of Glider configurations constrained by $\langle z\rangle_{\text {rel }}$, Eq. (2).

function of $\zeta$, following the Gaussian overlap approximation [3]. The resulting parametrization reads

$$
\frac{H_{i, j}^{\prime \prime}}{S_{i, j}^{\prime \prime}}=\frac{1}{2}\left(H_{i, i}+H_{k, k}\right)+B \zeta_{i, j}^{2} .
$$

Here $B$ is an introduced parameter. It is estimated from the corresponding known elements in $H$ and $S$. Alternatively, $B$ can be deduced from the kinetic Hamiltonian operator in the final state. The agreement between the two ways of estimating it gives a check on the reliability of the overall methodology.

There is a technical problem in calculating the Hamiltonian matrix element between the initial state and the continuum wave function, $\langle i|H| f\rangle$ in the FGR. The FGR requires that the initial state to be rigorously orthogonal to the continuum. This is certainly the case if the GCM configurations are HF eigenstates of different $K$ partitions. However, the code to compute $H_{i j}$ makes use of the Balian-Brezin contraction formula [15] which requires the two wave functions to have a finite overlap. ${ }^{3}$ Since we actually use the HFB machinery with some residual pairing, there is no difficulty calculating matrix elements between configuration connected by pairing. Thus one can use the code as is for those matrix elements. We deal with the nonzero overlaps by explicit orthogonalization as described in the Appendix.

\section{GLIDER IN THE CONTINUUM}

Configurations with the $K$ partition called "Glider" in Refs. [2,17] arise along a GCM-generated path for the fission of ${ }^{236} \mathrm{U}$. Glider is barely unstable with respect to fission, so it makes a good example for the construction of a continuum channel. Several characteristics of the Glider scission path are shown in Figs. 1-3. The configurations in the path are constrained only by the relative coordinate and the number fluctuation. The plot of $N_{\text {neck }}$ in Fig. 1 shows that the scission takes place rather suddenly near $z_{\text {rel }} \approx 16.75 \mathrm{fm}$. Figure 2 shows the mass quadrupole moment as a function of $z_{\text {rel }}$. It

\footnotetext{
${ }^{3}$ One may use instead the pfaffian based formula of Ref. [16] which is well defined in this case.
} 


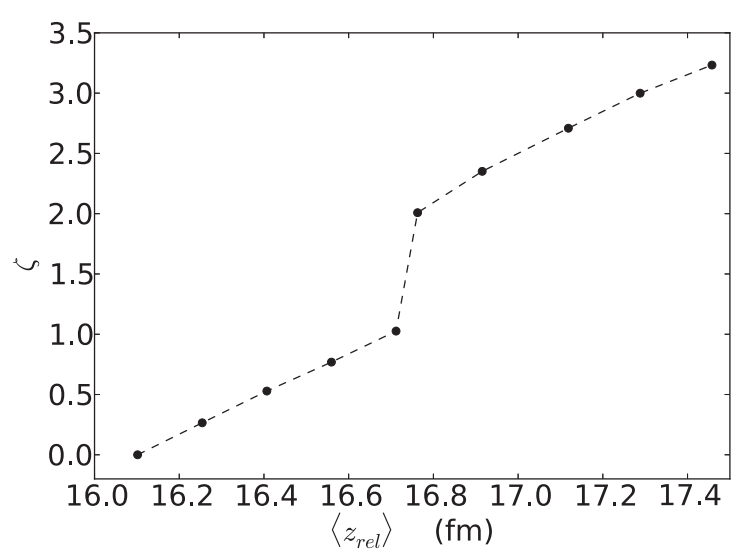
16.1 .

FIG. 3. Overlap distance $\zeta$ along the elongation path from $z_{\text {rel }}=$

varies nearly linearly with $z_{\text {rel }}$ for the range of separations shown in the figure, with only a slight offset at the scission point.

Figure 3 plots the overlap distance $\zeta$ [Eq. (10)] along the scission path between the coordinates $z_{\text {rel }}=16.1$ and $17.4 \mathrm{fm}$. One sees that there is a large jump at the scission point, showing that the overlaps of the configurations on each side is much smaller than between neighboring configurations elsewhere along the chain. Typical overlaps of the neighboring configurations marked by circles are in the range $0.85-0.95$, while it is only 0.38 across the scission point.

The HF energies along the scission path are shown in Fig. 4. The scission point is marked with an arrow. There one can see a small offset and change in slope. Beyond the scission point the slope of the curve should be largely determined by the Coulomb force between the two fragments. The red curve shows their Coulomb interaction, offset vertically to facilitate the comparison with the slope of the energy curve. The good agreement is promising for the method but could be somewhat misleading in view of the neglect of the nuclear interaction

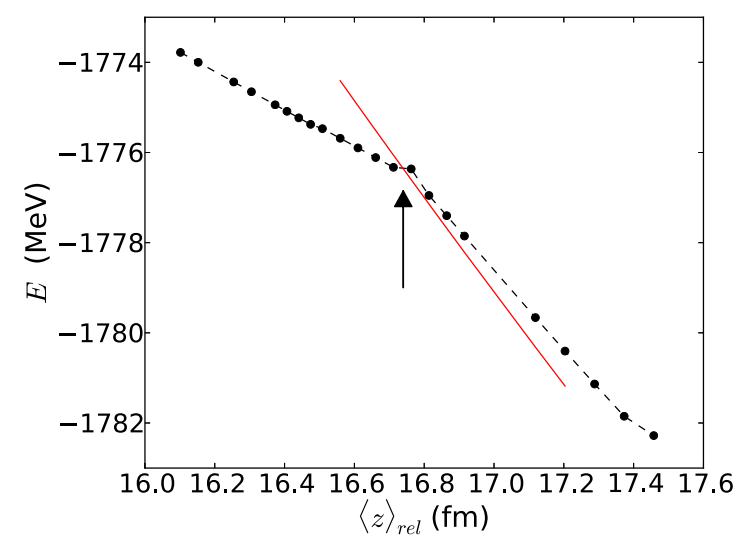

FIG. 4. Hartree-Fock energy as a function of the separation between centers of mass of the two nascent fragments. The red line shows their Coulomb interaction approximated as $V_{c}=$ $e^{2} Z_{L} Z_{R} / z_{\text {rel }}+C$ where $C$ is an offset to cross the energy curve at the scission point, marked by an arrow.

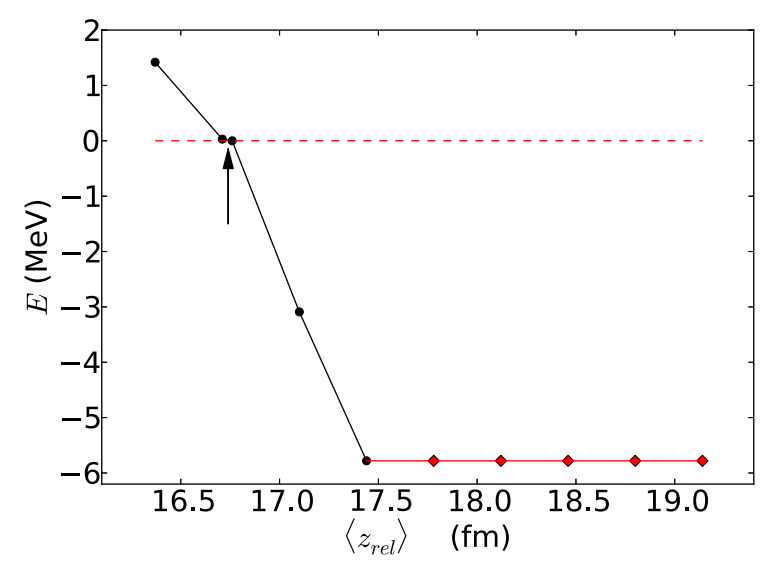

FIG. 5. Energies of configurations used to build the continuum wave functions. Black circles are GCM-constrained Glider configurations; arrow marks the scission point between the second and third state. Red diamonds are simulated configurations for large separations, characterized by $S$ and $H$ matrix elements as described in Eq. (12) and (13). The energy scale is with respect to the HF energy of the second configuration.

and the shape dependence of the Coulomb field. However, for well-separated fragments the energy nicely follows Coulomb law [18].

To construct the truncated continuum wave function we start with five glider configurations, composed of two prescission configurations and three just beyond the scission point. The farther two are spaced at intervals of $\Delta z_{\text {rel }}=0.34 \mathrm{fm}$ from the first postscission configuration. Except for the two on either side of the scission point, the overlaps of neighboring configurations are about 0.7 . This basis is augmented by $N_{\text {add }}=5$ more states at larger separations, with matrix elements defined as discussed in the previous section. The positions and energies of the basis states are shown in Fig. 5. For completeness, the positions, energies, and leading offdiagonal matrix elements are listed in Table I.

TABLE I. The test states in the space to approximate the continuum Glider wave function. The five configurations in the upper part of the table are obtained by the GCM minimization of the Gogny D1S energy functional. The lower five configurations are obtained by extrapolating the GCM matrix elements as described in the text. The last column shows the amplitudes of the fifth eigenstate in the spectrum.

\begin{tabular}{cccccr}
\hline \hline Source & $z_{\text {rel }}(\mathrm{fm})$ & $E(\mathrm{MeV})$ & $S_{i, i+1}$ & $H_{i j} / S_{i j}$ & \multicolumn{1}{c}{$a_{n}$} \\
\hline GCM & 16.37 & -1774.94 & 0.73 & -1776.94 & -0.24 \\
& 16.71 & -1776.32 & 0.38 & -1780.45 & -0.50 \\
& 16.76 & -1776.36 & 0.68 & -1778.45 & -0.21 \\
& 17.10 & -1779.29 & 0.72 & -1782.14 & 0.08 \\
\multirow{4}{*}{ Added } & 17.44 & -1782.14 & 0.72 & -1783.57 & 0.58 \\
& 17.77 &, &, &, & -0.46 \\
& 18.11 &, &, &, & -0.15 \\
& 18.45 &, &, &, & -0.08 \\
& 18.79 &, &, &, & 0.77 \\
& 19.13 &, & - & - & -0.62 \\
\hline \hline
\end{tabular}




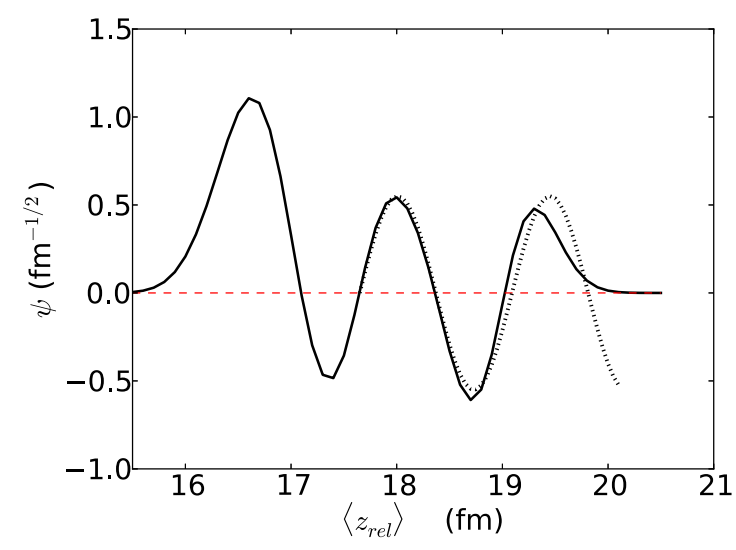

FIG. 6. Coordinate space wave function $\psi\left(z_{\text {rel }}\right)$ for the fifth eigenstate at $E_{5}=-1776.4 \mathrm{MeV}$ in the GCM spectrum. The fit to $A$ in the asymptotic wave function $A \sin \left(k z_{\text {rel }}+\delta\right)$ is shown as the dotted line in the range $z_{\text {rel }}=17.5-18.5$.

After diagonalizing the Hamiltonian, the eigenstate having energy closest to the initial state is taken as the continuum wave function of interest. For our test case here, we take the fifth state in the 10-dimensional space, with an eigenenergy of $E_{c}=-1776.40 \mathrm{MeV}$. This is close to that of the two configurations near the scission. The amplitudes $a_{5, n}$ of the eigenfunction are shown in the last column of Table I. The quality of the relative coordinate wave function can be assessed from its explicit dependence on $z_{\text {rel }}$. The conversion to $\psi\left(z_{\text {rel }}\right)$ is carried assuming that all configurations have the same Gaussian distribution [Eq. (9)]. From the overlap of adjacent configurations [Eq. (8)] we determine the parameter $s$ to be $0.3 \mathrm{fm}$. This is convoluted with the GCM amplitudes given in Table I to give the wave function $\psi$ shown in Fig. 6. The wave function computed this way comes out properly normalized,

$$
\int\left|\psi\left(z_{\text {rel }}\right)\right|^{2} d z_{\text {rel }}=1
$$

Next we compare with the Schrödinger wave function for the relative coordinate, which is just a plane wave for $z_{\text {rel }}>17.44 \mathrm{fm}$. A fit of the form $\psi(z)=A \sin (k z+\delta)$ is shown as the dotted line in Fig. 6. Its parameters are $k=$ $4.36 \mathrm{fm}^{-1}$ and $A=0.55 \mathrm{fm}^{-1 / 2}$. This is to be compared with the energy to be expected for a plane wave at that momentum in a flat potential. The energy of the $k=0$ wave function is lower than the diagonal energy of a GCM configuration $\left(E_{B}=-1782.14 \mathrm{MeV}\right)$ due to the kinetic energy of the GCM wave packet in the relative coordinate. The energy offset from Eq. (A4) of Ref. [5] is

$$
E_{0}=\frac{\hbar^{2}}{4 M_{\mathrm{red}} s^{2}},
$$

which evaluates to $2.05 \mathrm{MeV}$ with the parameters of our system: $s=0.30 \mathrm{fm} ; \hbar^{2} / M_{\text {red }}=\hbar^{2}\left(A_{L}+A_{R}\right) / m A_{L} A_{R} ; A_{R}=$ 136 and $A_{L}=100$. The kinetic energy evaluated from the GCM diagonalization is $T_{5}=E_{5}-E_{B}+E_{0}=7.9 \mathrm{MeV}$. This is somewhat larger than the kinetic energy for free particles at the same momentum, $T_{\text {free }}=\hbar^{2} k^{2} / 2 M_{\text {red }}=6.8 \mathrm{MeV}$. This can be interpreted as an (unphysical) effective mass $M^{*} / M=$ $6.8 / 7.9=0.87$. In the early literature, the calculation of the inertial masses by the GCM was shown to be a challenging problem, in general requiring a double projection [19]. However, as noted in Ref. [19] the second projection is unnecessary if the GCM wave function factorizes into an internal part multiplied by a function of the center-of-mass coordinate. Indeed, this one of the crucial assumptions [Eqs. (8) and (9)] in our treatment of the continuum channels. Unfortunately, it is not clear how to improve the present approach if the assumption is badly violated. In any case, the good fit of the sinusoidal wave function and the fair reproduction of the inertial mass is encouraging to use the GCM/CI framework for rough estimates of decay widths.

\section{DECAY WIDTH EXAMPLES}

We start with example of a decay through diabatic dynamics, namely the decay of the Glider configuration at $z_{\text {rel }}=$ $16.71 \mathrm{fm}$ to the Glider continuum. For this case, the decay width is large enough to assess the mixing directly from the eigenstates in a fairly small space. The strength function $P_{i}$ for a configuration in the spectrum of eigenfunctions is simply its probability as a function of the eigenenergies or a state label for the eigenfunctions. In the GCM basis this is given by

$$
P_{i}(\alpha)=\sum_{n}\left(S_{i, n} a_{\alpha, n}\right)^{2},
$$

where $i$ is the configuration of interest and $\alpha$ labels the eigenstates. This formula satisfies the expected normalization $\sum_{\alpha} P_{i}(\alpha)=1$. In the orthonormal basis the probability is given by

$$
P_{i}(\alpha)=\langle\tilde{i} \mid \tilde{\alpha}\rangle^{2} .
$$

As mentioned, we take the initial state to be the Glider configuration at $z_{\text {rel }}=16.71$. The space is augmented with three other GCM configurations at $z_{\text {rel }}=16.76,17.10$, and $17.44 \mathrm{fm}$ as well 20 configurations constructed with Eqs. (12) and (13) like the ones in the lower lines of Table I. The resulting strength function is shown by the vertical bars in Fig. 7. There is a considerable spread of the strength among the three eigenstates near $E=-1776 \mathrm{MeV}$. To assign a decay width we first smooth $P_{i}$ by convoluting the discrete strengths $P_{i}(\alpha)$ with a Gaussian spreading functions. Then a decay width can be defined as the full width of the smoothed strength function at half maximum, $\Gamma_{\mathrm{FWHM}}$. The extracted value from the figure is

$$
\Gamma_{\mathrm{FWHM}} \approx 3 \mathrm{MeV} .
$$

As a check on the modified FGR, we also determined the width by that method. Figure 8 shows the off-diagonal Hamiltonian matrix elements in the first row of Eq. (A6). The product is $v^{2}=0.45 \mathrm{MeV}^{2}$ at $E_{\beta} \approx E_{i}$; the spacing of energies there is $\Delta E \approx 1.3 \mathrm{MeV}$. Inserting these into Eq. (A7) the estimated decay width is

$$
\Gamma_{\mathrm{FGR}}=2.2 \mathrm{MeV} .
$$




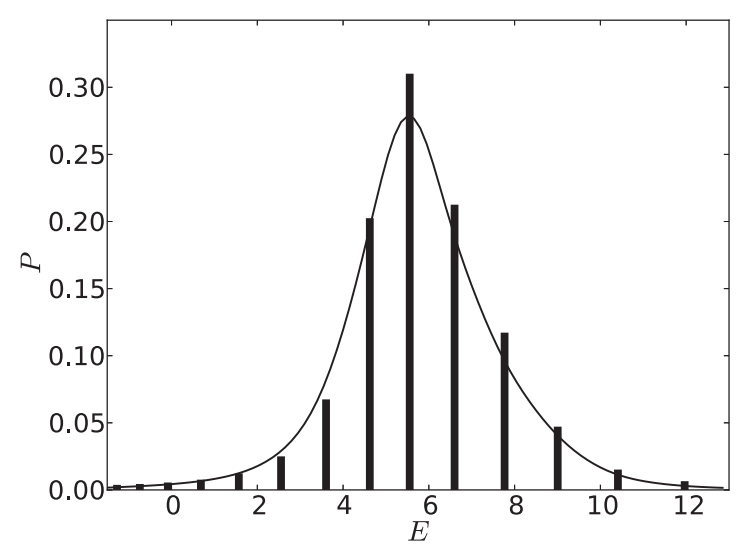

FIG. 7. The strength function for the Glider configuration at $z_{\text {rel }}=16.71 \mathrm{fm}$ dissolving into the discretized continuum eigenstates. The horizontal axis is the energy of the eigenstates $E_{\alpha}$ in $\mathrm{MeV}$ with respect to the flat potential in the external region. See text for construction of the discretized basis. The curve shows the strength function smoothed by convoluting the discretized strength function with Gaussian envelopes.

The agreement is only fair, but one must remember that the procedure to determine the width from the discrete-basis strength function was somewhat ad hoc.

To see how these numbers depend on the numerical parameters Table II shows the effect on the calculated quantities under changes of the number of continuum states and their spacing. From the table it appears that a mesh spacing of $0.34 \mathrm{fm}$ in the relative coordinate with 10 or more states in the continuum is adequate to compute the effective mass to about $10 \%$ uncertainty. The computed value is lower that the physical by about $10 \%$ as well. This impacts the reliability of the calculated widths by that amount. Turning to the width estimates in the table, one sees that ones calculated by the FGR cluster closer together than the ones calculated from the shape of the strength function. Also, one can see that the value we obtained in Sec. IV [Eq. (18)] is an outlier. The other

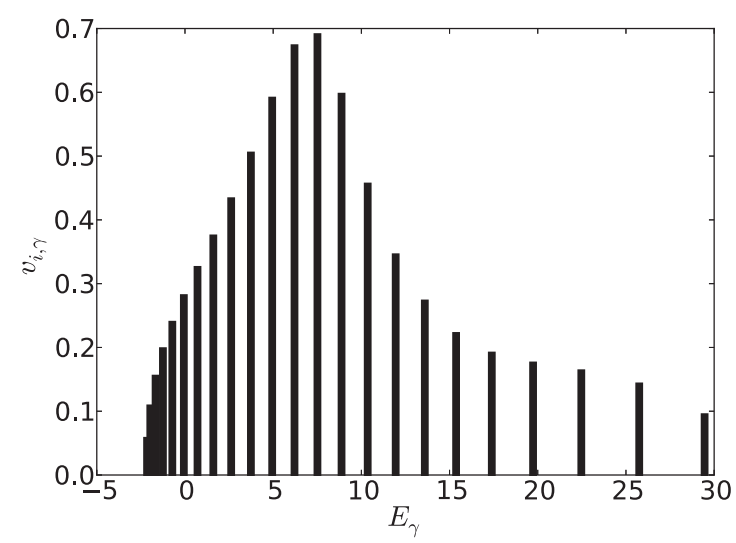

FIG. 8. Off-diagonal matrix elements between the initial state and final states in the $H^{\gamma}$ matrix. The horizontal axis is the energy of the eigenstates $E_{\gamma}$ with respect to the flat potential in the external region. Units are $\mathrm{MeV}$ for both axes.
TABLE II. Effect on derived quantities of changes in numerical parameters for calculating the continuum wave function. For the $N_{\text {add }}$ column, the first number corresponds to the effective mass calculation and the second number to the width calculations. The first row summarizes the results described in Secs. III and IV. The percentage changes in the derived quantities are shown in the fourth to sixth columns of the rows below that.

\begin{tabular}{lccccc}
\hline \hline & $\begin{array}{c}\Delta z_{\text {rel }} \\
(\mathrm{fm})\end{array}$ & $N_{\text {add }}$ & $M^{*} / M$ & $\begin{array}{c}\Gamma_{\mathrm{FWHM}} \\
(\mathrm{MeV})\end{array}$ & $\begin{array}{c}\Gamma_{\mathrm{FGR}} \\
(\mathrm{MeV})\end{array}$ \\
\hline Base & 0.34 & $5 / 24$ & 0.87 & 3 & 2.2 \\
$\mathrm{a}$ & 0.34 & $10 / 36$ & 0.91 & 2.3 & 2.6 \\
$\mathrm{~b}$ & 0.50 & $5 / 36$ & 0.79 & 1.9 & 2.3 \\
\hline \hline
\end{tabular}

estimates give a range of widths from 1.9 to $2.6 \mathrm{MeV}$, a spread of $\pm 15 \%$ around the mean.

Next we treat two cases where the decaying configuration is in a different $K$ partition from Glider. In Ref. [17] we found that Glider was populated from the bound configuration Buenavista by two pair jumps. The orbitals involved are shown in Table III. The intermediate configuration labeled "A" and "B" will be treated as the initial states for the FGR width calculations. The coordinate $z_{\text {rel }}$ of A and B is set to $16.71 \mathrm{fm}$, the same as the coordinate of the decaying configuration in the previous paragraph. Besides $\mathrm{A}$ or $\mathrm{B}$, the space in the calculation includes all of the GCM states in Table I together with 20 added configurations in the continuum. The results are shown as the last column in Table III. One should be cautious in making any quantitative interpretation of these widths, due to the numerous approximations made to obtain them. But we believe that two conclusions can be drawn already from the three examples. The first is that the widths from diabatic dynamics are larger than those from pairing Hamiltonian by two orders of magnitude or more. The other conclusion is that we should expect large fluctuations in the widths associated with pairing interaction.

\section{DISCUSSION}

We have demonstrated how the GCM framework can be applied to a fully quantum calculation of the final step in nuclear fission, namely the rupture of the neck joining the two nascent fragments. There do not seem to be major obstacles to pursuing this approach to the point where one can estimate average or total decay widths of the very elongated prescission configurations. We presented here a calculated decay rate for a

TABLE III. Transitions from Buenavista to Glider via intermediate configurations $\mathrm{A}$ and $\mathrm{B}$.

\begin{tabular}{lccc}
\hline \hline $\begin{array}{l}\text { Initial } \\
\text { Configuration }\end{array}$ & Pair jump & $\begin{array}{c}\text { Final } \\
\text { configuration }\end{array}$ & $\Gamma_{\mathrm{FGR}}$ \\
\hline Buenavista & $(1 / 2)_{p}^{2} \rightarrow(3 / 2)_{p}^{2}$ & $\mathrm{~A}$ & \\
Buenavista & $(1 / 2)_{n}^{2} \rightarrow(9 / 2)_{n}^{2}$ & $\mathrm{~B}$ & \\
A & $(1 / 2)_{n}^{2} \rightarrow(9 / 2)_{n}^{2}$ & Glider & $5 \mathrm{keV}$ \\
B & $(1 / 2)_{p}^{2} \rightarrow(3 / 2)_{p}^{2}$ & Glider & $60 \mathrm{keV}$ \\
\hline \hline
\end{tabular}


configuration that undergo scission by diabatic dynamics and for two others configuration that required a residual interaction to reach the same decay channel. We hesitate to draw general conclusions from just these three examples, especially in view of the large fluctuations in residual interaction matrix elements exhibited in Table I and also large variations in the overlaps of the configurations contributing to diabatic decay paths [2].

It is expected that the diabatic dynamics would dominate when permitted. For example, the collective masses calculated by cranking or in other approximations have much larger contributions from the pairing than the mean-field interaction. But finding two to three orders of magnitude difference in their contribution to decay widths is surprisingly large. One effect that could boost the pairing-assisted decays is coherence of the pairing field in the HFB condensate. However, that effect is diminished when the initial wave function contains quasiparticle excitations that suppress the pairing condensate. We still don't have a clear picture of how much thermal excitation energy is present at the scission point, but the observed presence of odd-even staggering in the fragment charge distributions indicates that some pairing correlations remain.

Experimentally, the finding [20] that there are no systematic fluctuations in the fission cross section on a 1-keV energy scale indicates that the average total decay rates mediated by the residual interaction should be considerably larger. All three of the estimated partial widths were indeed much larger, so the theory is at least consistent with the observations on this point.

It is also interesting to compare with rates found in the time-dependent Hartree-Fock-Bogoliubov calculations of Ref. [21]. In that work it was seen that the early shape changes to very elongated shapes evolved steadily to the scission point, but the nucleus stops there for a length of time of the order of $10000 \mathrm{fm} / c$ before scission occurs. Converting that time to a decay width gives $\Gamma \approx \hbar / \tau \approx 20 \mathrm{keV}$, which is between the calculated decay rates of the $\mathrm{A}$ and $\mathrm{B}$ configurations into the Glider channel. Thus, our microscopic calculation offers some confirmation of the TDHFB dynamics.

A long-term goal of fission decay width theory is to calculate not only total widths but branching ratios as well. If the GCM-based theory could be developed to a point where a representative sample of final continuum channels can be constructed, then it would be possible to estimate branch ratios into the different channels and thus fluctuations in all observables. A good example is the odd-even staggering in mass distributions. The overall mass yield curves very likely depend mainly on statistical dynamics up to populating prescission configurations, but the final division including pair breaking requires understanding the scission dynamics. Another example is the total kinetic energy distribution, which is determined by the access to different exit channels. It might be the case that multiple exit channels compete in the decay of a prescission configuration. In our first study [17], we found that the prescission configuration Buenavista could connect with two $K$ partitions, Glider and Bobsled, which exit at quite different kinetic energies.
However, there are many problems to be overcome before the theory can be easily applied to representative samples of configurations. One shortcoming of the present formulation is the lack of collective flow in the GCM parameter space. The motion of the fragments in the final state is present in the model space within certain limits. However, to treat wave functions at fragment separations more than a fermi or so the kinetic energy would have to be included. In the GCM, this could be achieved by a placing a constraint on the momentum operator. Operators including currents are also important to assess the role of collective flow in prescission configurations.

Another problem is a technical one. Namely, the procedure we followed to calculate the residual interaction between configurations of $K$ partitions is specific to the pairing interaction, relying as it does on the nonzero overlaps of the HFB wave functions. The nonpairing residual interaction is responsible for pair-breaking and increasing (or decreasing) the internal excitation energy by creating or annihilating quasiparticles. It is certainly achievable to treat residual interactions of more general form, but this requires new coding, employing the general algorithm of Ref. [16] instead of the more traditional one of Ref. [15].

Another important shortcoming of the method as carried out here is absence of quasiparticle excitations in the GCM wave functions. Very likely a significant fraction of the excitation energy in the primordial fission fragments is in the form of particle-hole excitations above the base GCM configuration [22]. It is crucial to know the internal excitation energy of the nascent fragment to model the subsequent decays emitting neutrons and $\gamma$ rays.

Finally, we comment on other fully quantum approaches to fission dynamics. The time-dependent HFB approximation has been shown to be computationally feasible [21] and interesting results have been obtained from it: The dynamics is overdamped in the elongation phase and there is a long delay at the scission point. But as a mean-field approximation, the HFB can only give average behavior and not fluctuations. Another approach closer to ours is that of Ref. [23]. They consider a large number of GCM configurations in the HFB approximation and derive a Schrödinger equation for collective GCM variables. This approach was found to gives reasonable fluctuations in the mass yields. But it may not be so well suited for other quantities, such as the role of quasiparticle excitations [22]. And the particular dynamics at the scission point seems to us to be beyond the reach of approaches based on collective shape variables.

\section{ACKNOWLEDGMENTS}

We thank W. Younes and U. Mosel for discussions and again W. Younes for contributions to computational aspects of the present approach. The work of L.M.R. was partly supported by Spanish MINECO Grants No. FPA2015-65929 and No. FIS2015-63770.

\section{APPENDIX: STRENGTH FUNCTION AND DECAY WIDTH IN THE GCM BASIS}

The strength function $P_{i}(E)$ of a configuration $i$ mixing with other configurations is fundamental to the derivation of 
decay widths. $P$ is simply given by the probability of the state $i$ in the eigenstates $\alpha$ of the Hamiltonian of the full configuration space,

$$
P_{i}(E)=\sum_{\alpha}\langle\tilde{i} \mid \tilde{\alpha}\rangle^{2} \delta\left(E-E_{\alpha}\right),
$$

where the tilde indicate states defined in the orthonormal basis. ${ }^{4}$ Alternately, the Hamiltonian can be diagonalized directly in the GCM basis as the generalized eigenvalue equation

$$
S^{-1} H|\alpha\rangle=E_{\alpha}|\alpha\rangle \text {. }
$$

Here the $|\alpha\rangle$ are normalized by

$$
\left\langle\alpha|S| \alpha^{\prime}\right\rangle=\sum_{n, n^{\prime}} a_{n, \alpha^{*}}^{*} S_{n, n^{\prime}} a_{n, \alpha^{\prime}}=\delta_{\alpha, \alpha^{\prime}}
$$

Then the strength function is computed as

$$
P_{i}(E)=\sum_{\alpha}\langle i|S| \alpha\rangle^{2} \delta\left(E-E_{\alpha}\right) ;
$$

this formulation also satisfies the sum rule $\int P_{i}(E) d E=1$.

When the decays into the continuum is weak, the strength function approaches a Breit-Wigner shape $P \sim$ $1 /\left[\left(E-E_{i}\right)^{2}+(\Gamma / 2)^{2}\right]^{2}$ corresponding to an exponential decay $e^{-\Gamma t}$ in the time domain. Here the FGR can be applied to determine $\Gamma$. Since Eq. (1) assumes orthonormality of initial and final states, the safest way to evaluate the FGR is in an orthonormal basis. This requires several transformations from the original GCM representation. The first step is to convert the vectors to the tilde representation; the resulting

\footnotetext{
${ }^{4}$ The transformation matrix between the GCM and the orthonormal basis is $S^{1 / 2}$.
}

Hamiltonian matrix $H_{\alpha, \alpha^{\prime}}^{\alpha}$ is Hermitean. The next transformation is to tridiagonalize $H^{\alpha}$ by the Lanczos method using the state $|\tilde{i}\rangle$ as the pivot. This yields the tridiagonal matrix $H^{\beta}$ with basis vectors $|\tilde{i}\rangle,\left|\beta_{1}\right\rangle,\left|\beta_{2}\right\rangle, \ldots$. All the states are now orthogonal, but to apply the FGR we still need to diagonalize the Hamiltonian in the $\beta$ subspace. That is carried out by the transformation matrix

$$
U=\left[\begin{array}{cc}
1 & 0 \\
0 & U^{\prime}
\end{array}\right]
$$

where $U^{\prime}$ is the transformation matrix to diagonalize $H^{\beta}$ in the $\beta$ subspace. The final form of the Hamiltonian is

$$
H^{\gamma}=\left[\begin{array}{cc}
E_{i} & v_{i, \gamma} \\
v_{i, \gamma^{\prime}}^{T} & E_{\gamma} \delta_{\gamma, \gamma^{\prime}}
\end{array}\right] .
$$

The matrix elements needed to apply the FGR are the offdiagonal ones in the first row. In principle the space should be large with a high-enough density of final states to calculate an average $|v|^{2}=\overline{\left|v_{i, \gamma}\right|^{2}}$ over some interval. Also, the spacing of continuum states should be uniform enough to assign an average spacing $\Delta E$. Then the FGR can be evaluated as

$$
\Gamma=\frac{2 \pi}{\Delta E}|v|^{2} .
$$

Since the size of the spaces is rather small in our examples we have taken $|v|^{2}$ from the matrix element to the state $\gamma$ that is closest in energy to the initial state. The corresponding energy spacing was taken to be $\Delta E=\left(E_{\gamma+1}-E_{\gamma-1}\right) / 2$. This is very approximate but seems adequate to estimate the orders of magnitude of the decay widths.
[1] N. Schunck and L. M. Robledo, Rep. Prog. Phys. 79, 116301 (2016).

[2] G. F. Bertsch, W. Younes, and L. M. Robledo, Phys. Rev. C 100, 024607 (2019).

[3] M. Bender, P.-H. Heenen, and P.-G. Reinhard, Rev. Mod. Phys. 75, 121 (2003).

[4] L. M. Robledo, T. R. Rodríguez, and R. R. Rodríguez-Guzmán, J. Phys. G: Nucl. Part. Phys. 46, 013001 (2019).

[5] G. F. Bertsch and W. Younes, Ann. Phys. 403, 68 (2019).

[6] E. Caurier, G. Martinez-Pinedo, F. Nowack, A. Poves, and A. P. Zuker, Rev. Mod. Phys. 77, 427 (2005).

[7] G. F. Bertsch (unpublished).

[8] M. Warda, J. L. Egido, L. M. Robledo, and K. Pomorski, Phys. Rev. C 66, 014310 (2002).

[9] L. M. Robledo (unpublished).

[10] J. Dobaczewski, M. V. Stoitsov, W. Nazarewicz, and P.-G. Reinhard, Phys. Rev. C 76, 054315 (2007).

[11] T. Duguet, M. Bender, K. Bennaceur, D. Lacroix, and T. Lesinski, Phys. Rev. C 79, 044320 (2009).

[12] L. M. Robledo, J. Phys. G 37, 064020 (2010).
[13] P. Bonche, J. Dobaczewski, H. Flocard et al., Nucl. Phys. A 510, 466 (1990).

[14] See Supplemental Material at http://link.aps.org/supplemental/ 10.1103/PhysRevC.100.044606 for data files corresponding to figures and the table, scripts to generate them, and the code GCM [9] to calculate interaction matrix elements.

[15] R. Balian and E. Brezin, Nuovo Cim. B 64, 37 (1969).

[16] G. F. Bertsch and L. M. Robledo, Phys. Rev. Lett. 108, 042505 (2012).

[17] G. F. Bertsch, W. Younes, and L. M. Robledo, Phys. Rev. C 97, 064619 (2018).

[18] M. Warda and L. M. Robledo, Phys. Rev. C 84, 044608 (2011).

[19] R. Peierls and D. Thouless, Nucl. Phys. 38, 154 (1962).

[20] G. F. Bertsch, D. Brown, and E. D. Davis, Phys. Rev. C 98, 014611 (2018).

[21] A. Bulgac, P. Magierski, K. Roche, and I. Stetcu, Phys. Rev. Lett. 116, 122504 (2015).

[22] R. Bernard, H. Goutte, D. Gogny, and W. Younes, Phys. Rev. C 84, 044308 (2011).

[23] H. Goutte, J.-F. Berger, P. Casoli, and D. Gogny, Phys. Rev. C 71, 024316 (2005). 